\title{
DINÁMICA DEL EMPRENDIMIENTO Y EL DESEMPLEO EN LA PATAGONIA CHILENA
}

\author{
SERGIO ZUNIGA-JARA*, SERGIO SOZA-AMIGO** \& \\ KARLA SORIA-BARRETO
}

\begin{abstract}
RESUMEN $^{1}$
El objetivo de este trabajo ha sido investigar la existencia de relaciones estadísticas entre la actividad emprendedora y la creación de empleo en las principales comunas de la Patagonia Chilena (Valdivia, Osorno, Puerto Montt, Coyhaique y Punta Arenas). Para esto se utilizan datos del periodo 2009 al 2012. Como resultado, se encuentra evidencia de que sólo en el periodo 2011 a 2012 el empleo efectivamente puede ayudar a predecir el nivel de creación de empresas. Esta relación es conocida como "efecto Refugio", el que puede asociarse con un emprendimiento "por necesidad". No fue posible encontrar evidencia a favor de la hipótesis de que el crecimiento económico es el que genera nuevas oportunidades y con ello una disminución del desempleo ("efecto Oportunidad"). Los resultados son relevantes por cuanto a la fecha no se han realizado en la Patagonia Chilena estudios o encuestas sobre fuentes primarias que permitan caracterizar el emprendimiento.
\end{abstract}

PALABRAS CLAVE: "Efecto Refugio", "Efecto Oportunidad”, Emprendimiento, Desempleo.

\section{ENTREPRENEURSHIP AND UNEMPLOYMENT DYNAMIC IN THE PATAGONIA OF CHILE}

\begin{abstract}
The goal of this work is to research the existence of statistical relations between entrepreneurial activity and the creation of employment at the main communes in Chilean Patagonia (Valdivia, Osorno, Puerto Montt, Coyhaique and Punta Arenas). Data from the 2009-2012 period were used. As a result, evidence was found indicating that only in the period 2011-2012 the employment can effectively help to predict the creation of new business. This relationship is known as "Refuge effect", which can be associated to "entrepreneurship due to necessity". No evidence was found to support the hypothesis of

* Escuela de Ciencias Empresariales, Universidad Católica del Norte, Coquimbo, Chile. sz@ucn.cl.

* Facultad de Ciencias Económicas y Jurídicas, Universidad de Magallanes, Punta Arenas, Chile. sergio.soza@umag.cl.

Escuela de Ciencias Empresariales, Universidad Católica del Norte, Coquimbo, Chile. ksoria@ucn.cl.

1 Se reconocen y agradecen los financiamientos entregados por Fondecyt por medio de la investigación "Relaciones Político Administrativas y su Funcionalidad para el Último Lustro en el Eje Longitudinal de la Vertiente de Barlovento de la Cordillera de Los Andes Norpatagónicos", Proyecto Número 1140936.
\end{abstract}


economic growth to be the generator of new opportunities and, thus, produce a diminution of unemployment ("Opportunity effect"). The results are relevant since, to this date, no studies or surveys have been conducted on primary sources in Chilean Patagonia to characterize entrepreneurship.

KEY WORDS: "refugee effect", "opportunity effect", entrepreneurship, unemployment, Patagonia.

\section{INTRODUCCIÓN}

A través de los últimos años, el emprendimiento ha ido adquiriendo cada vez mayor atención a nivel mundial, por cuanto se le considera como uno de los principales motores del crecimiento y del desarrollo económico. Implícitamente se considera entonces que el emprendimiento está fuertemente vinculado a la dinámica del empleo. Uno de los aspectos que hacen atractiva la relación desempleoemprendimiento son los diversos estudios que han demostrado una relación bi-variada positiva entre ambas. Sin embargo, hasta ahora se han dejado de lado dos aspectos relevantes para la Patagonia Chilena, lo cual es la motivación del presente estudio. Por un lado, se busca indagar si la evidencia empírica internacional acerca de esta relación se repite en éste vasto territorio y; por otra parte, si ante un incremento del desempleo se observa un aumento en las actividades y emprendimientos propios del "turismo" en las principales capitales comunales de esta. Esto último es relevante dadas las condiciones naturales que ofrece la Patagonia para desarrollar el mismo y la cantidad importante de Parques Nacionales que existen en éste territorio; en tal sentido, se revisará si el emprendimiento que se realiza en las principales comunas de la Patagonia, están más vinculados al sector turismo o hacia el resto de actividades. Con tal proxy se responderá si esas comunas muestran una tendencia hacia una vocación productiva que a futuro se pueda considerar como "comunas comprometidas con el turismo".

La relación entre emprendimiento y desempleo ha sido estudiada desde hace muchos años. Dos visiones opuestas de las fuerzas en juego son las siguientes:

a) Por una parte se espera que un aumento en las tasas de desempleo conduzcan a un incremento en las tasas de creación de empresas. Este es lo que se conoce como "efecto Refugio" (refugee o 'shopkeeper' effect) en el sentido de que el desempleo estimularía el emprendimiento con gran fuerza, o sería un catalizador para el crecimiento de nuevas empresas. Los emprendimientos que surgen por necesidad se basan en la necesidad de las personas de auto emplearse, muchas veces con cierta urgencia por conseguir ingresos económicos, dado que no consiguen empleo como dependientes. La figura 1 ilustra este efecto vinculando desempleo y emprendimiento con una flecha de derecha a izquierda. Algunas evidencias favorables a este resultado se encuentran en Reynolds et al. (1995); Evans et al. (1990); Blau (1987); Evans y Leighton (1990); Evans y Jovanovic (1989); y Blanchflower y Meyer (1994).

b) Una posición opuesta surge del llamado "efecto Oportunidad". En efecto en su libro probablemente más famoso, Schumpeter (1942) desarrolla la idea de que el desempleo es un fenómeno fundamentalmente friccional o de corto plazo, el que surge de un proceso de destrucción creativa cuando los factores de producción son reasignados de las unidades que están en contracción hacia las unidades económicas que están en proceso de expansión. Es decir, la "re-estructuración laboral" es un proceso natural que ocurre cada cierto tiempo cuando la economía se redirige hacia sistemas de producción más eficientes (figura 1). Si bien este concepto actualmente es atribuido a Schumpeter, los fundamentos de la noción de desempleo tecnológico se encuentran en Ricardo (1821). La evidencia empírica reciente apoyando esta hipótesis y detectando una relación inversa entre el desempleo y el nacimiento de nuevas empresas, la proporciona Audretsch (1995). En forma similar, Audretsch y Thurik (2000) encuentran que un aumento en el número de empresas reduce los niveles de desempleo.

Un mayor crecimiento económico global se traduce en altas tasas emprendimiento y, debido a las nuevas oportunidades que surgen como consecuencia de la expansión, en mayores niveles 


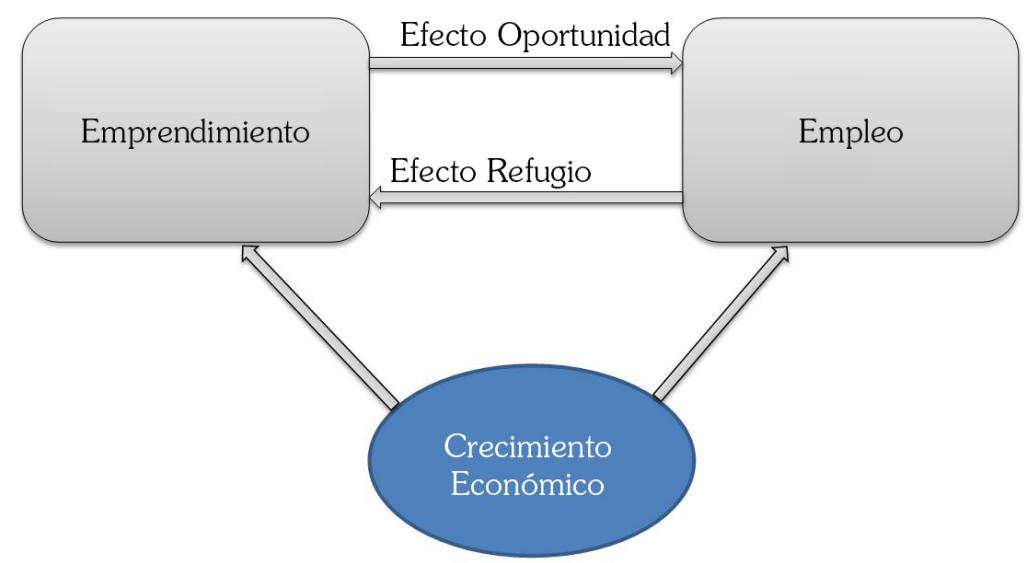

Fig 1. Efecto Oportunidad y Efecto Refugio como explicativas de la relación emprendimiento y desempleo. Fuente: Propia.

de empleo de acuerdo a Schumpeter. La visión opuesta indica que una expansión reduce el interés por emprender nuevos negocios, debido al boom existente los agentes económicos se encontrarían sin la necesidad de esto. En consecuencia, el crecimiento económico global es uno de los principales causantes de los efectos sobre el emprendimiento y el desempleo, tal como también se muestra en la Fig. 1.

Las relaciones anteriores también están influidas por los efectos que estas han tenido en el pasado o rezagos (Picot et al. 1998). A su vez, el tamaño de las empresas puede resultar ser relevante, debido a que para grandes empresas su contribución a la creación de empleos es mucho menor al ser la tasa de penetración o participación en el empleo de los nuevos emprendimientos muy baja, lo que puede obscurecer las contribuciones al empleo. También se debe señalar que algunos estudios no han encontrado evidencia significativa de ninguna de las hipótesis anteriores, como por ejemplo Carree (2001). Por su parte, Audretsch et al. en 2001 obtienen como válidas dos ecuaciones distintas y separan los efectos "Oportunidad" y "Refugio". Por su parte, en Thurik (2003), se llega a resultados poco precisos.

Sin embargo, esas discordancias no son nuevas, Storey ya en 1991, concluía que en los análisis de serie de tiempo el desempleo se encuentra positivamente relacionado con los índices de formación de empresas, mientras que a nivel de series con datos de cortes transversales o mixtos, los estudios parecen apoyar una relación inversa o negativa. Hasta el momento parece no existir una propuesta que reconcilien tales diferencias.

Adicionalmente los emprendedores parecen tener características distintas. Por una parte los negocios surgidos de la necesidad difícilmente logran prosperidad financiera o competitividad, por cuanto cuentan con menores recursos respecto a quienes emprenden por oportunidad. Entonces, el emprendedor por necesidad parece tener más edad que el emprendedor vocacional lo que se traduce en mayor aversión al riesgo, menos tolerancia al fracaso y menores habilidades propias del emprendedor. La menor educación formal, y los menores estudios y la mayor experiencia caracterizan al emprendedor por necesidad, junto con una visión generalmente de más corto plazo, y a un menor tamaño de sus empresas. A pesar de esto, de acuerdo a la Gibrat's Law el crecimiento de las empresas es independiente de su tamaño, lo que tiene como implicancia que la actividad económica global tiene un similar efecto en el empleo de empresas grandes y pequeñas.

El Informe Global Entrepreneurship Monitor (GEM, por Amorós \& Poblete, 2012) indica que Chile tiene una de las tasas de emprendimiento más altas del mundo, y que casi el $60 \%$ inicia un negocio por oportunidad y no por necesidad, mientras que sólo el $20 \%$ lo hace por no encontrar opción en el mercado laboral. Esto ubicaría a Chile por encima del promedio de la región, y en un nivel muy similar al comportamiento observado en los Estados Unidos. Para la Asociación de Emprendedores de Chile, el 
emprendimiento que proviene del quintil más rico es generalmente por oportunidad, mientras que en el quintil más pobre los emprendimientos tienden a ser por necesidad, y típicamente femeninos (jefas de hogar). Entonces, detrás de las altas tasas de emprendimiento de países pobres parecen esconderse emprendimientos por necesidad, mientras que en los países ricos se presenta una alta proporción de emprendimiento por oportunidad, y una bajísima tasa de emprendimiento por necesidad (Larroulet \& Ramírez, 2007). Similares resultados son reportados por Amorós y Echecopar (2008) y por Corfo (2013). Alguna evidencia a favor del efecto refugio en Chile es encontrado por Hinojosa et al. (2013) y por el Ministerio de Economía (2013) a través de su encuesta de microemprendimiento.

En base a la discusión anterior, la motivación de este estudio es explorar las relaciones existentes entre emprendimiento y desempleo en las principales capitales comunales de la Patagonia Chilena (Valdivia, Osorno, Puerto Montt, Coyhaique y Punta Arenas); esto es, indagar si la mayoría de los chilenos de esas zonas que emprenden lo hace porque ven una oportunidad en el mercado o, por una necesidad económica. Dos son las principales dificultades que se tienen para esto. En primer lugar el hecho de que la encuesta GEM de emprendimiento no se realiza en dichas regiones; y por otra parte, la escases de datos locales primarios. Para resolver esto, como explica en el apartado metodológico, se incluyen datos de varios años recientes para una serie de sectores económicos y para varias comunas de la Patagonia.

\section{METODOLOGÍA}

El enfoque de análisis de este trabajo se basa en matrices de transición y pruebas de independencia de Chi Cuadrado para la comparación de frecuencias observadas y esperadas, y a partir de estas concluir acerca de las relaciones intertemporales entre dos variables. Este procedimiento ha sido aplicado anteriormente por Altman et al. (1974); Alexander y Chervany (1980); Theobald (1981), entre otros autores. Para esto se asigna un número (clasificación) 1, 2 ó 3 a las diversas actividades económicas de cada comuna patagónica de acuerdo al nivel de creación de empresas y al nivel de desempleo de los tres años usados en el análisis. Si el dato observado en un año se ubica en el primer tercíl, entonces decimos que su nivel es bajo, en el segundo tercíl su nivel es medio, y en el tercer tercíl su nivel es alto (Tabla 1).

Para estudiar la dinámica de ambas variables y las hipótesis relacionadas se hacen tabulaciones cruzadas y se forman matrices de transición, contando el número de casos en que cierta clasificación en un período, se mantiene o no en el siguiente. En este sentido, las matrices de transición expresan la dinámica de las relaciones. En efecto, los elementos de la diagonal de las matrices contienen el número total de ciudades y actividades económicas que mantienen el mismo nivel relativo en ambos períodos. La Tabla 1 resume lo anterior, y muestra que la variable creación de empresas en el año t es identificada en tres rangos, al igual que la variable creación de empleo. Luego, ambas clasificaciones son asignadas a las filas o columnas de la matriz de transición, según lo requiera el análisis.

La primera hipótesis a probar será la existencia del efecto Oportunidad o de Emprendimiento por Oportunidad. Para esto se analiza para un determinado nivel de significancia, si una comuna patagónica que pertenece a un determinado portafolio o nivel de emprendimiento en un período (t), posee o no ese nivel de desempleo en el período siguiente $(\mathrm{t}+1)$ o incluso en el periodo subsiguiente $(t+2)$. Un Efecto Oportunidad implicaría que altos niveles de emprendimiento en ( $t$ ) tendrá un efecto significativo en la estructura de desempleo en algún periodo siguiente. La segunda hipótesis es la existencia de un Efecto Refugio. Para esto se analiza si la comuna que pertenece a un determinado portafolio o nivel de desempleo en un período ( $t)$, presenta un nivel distinto de emprendimiento en el período siguiente $(t+1) \mathrm{o}$ en $(t+2)$. Finalmente, un Efecto Refugio implicaría cambios significativos en los niveles de emprendimiento posteriores.

Para determinar si existe una asociación significativa entre las dos variables se utiliza la prueba Chi cuadrado, donde la hipótesis de independencia se aplica sobre variables categóricas, que para el caso estudio son los portafolios organizados en matrices como se indicó en la Tabla 1. 
Tabla 1. Criterios de clasificación y estructura básica de las matrices de transición.

\begin{tabular}{|c|c|c|c|c|}
\hline $\begin{array}{c}\text { Creación de Empresas } \\
\text { año "t" }\end{array}$ & Rango & & $\begin{array}{l}\text { Creación de Empleo } \\
\text { en el año "t" }\end{array}$ & Rango \\
\hline Crecimiento bajo & $\begin{array}{l}\text { Primer tercíl } \\
\text { (Bajo) }\end{array}$ & \multirow{3}{*}{$\downarrow$} & Creación de empleo bajo & $\begin{array}{l}\text { Primer tercíl } \\
\text { (Bajo) }\end{array}$ \\
\hline Crecimiento medio & $\begin{array}{l}\text { Segundo tercíl } \\
\text { (Medio) }\end{array}$ & & Creación de empleo medio & $\begin{array}{l}\text { Segundo tercíl } \\
\text { (Medio) }\end{array}$ \\
\hline \multirow[t]{4}{*}{ Crecimiento alto } & $\begin{array}{l}\text { Tercer tercil } \\
\text { (Alto) }\end{array}$ & & Creación de empleo alto & $\begin{array}{l}\text { Tercer tercíl } \\
\text { (Alto) }\end{array}$ \\
\hline & \multicolumn{3}{|c|}{ Matriz de Transición } & \\
\hline & & $\begin{array}{c}\text { Medio en } \\
\quad(t+1)\end{array}$ & $\begin{array}{l}\text { Alto en } \\
(t+1)\end{array}$ & \\
\hline & $\begin{array}{l}\text { Bajo en }(t+1) \\
\text { Medio }(t+1) \\
\text { Alto en }(t+1)\end{array}$ & & & \\
\hline
\end{tabular}

Establecido lo anterior, se plantean las seis hipótesis nulas a probar:

$\mathrm{H}_{01}$ : El emprendimiento en ( $\mathrm{t}$ ) no ayuda a predecir el desempleo en $(t+1)$

$\mathrm{H}_{02}$ : El emprendimiento en ( $\mathrm{t}$ ) no ayuda a predecir el desempleo en $(\mathrm{t}+2)$

$\mathrm{H}_{03}$ : El emprendimiento en $(\mathrm{t}+1)$ no ayuda a predecir el desempleo en $(\mathrm{t}+2)$

$\mathrm{H}_{04}$ : El desempleo en ( $\mathrm{t}$ ) no ayuda a predecir el emprendimiento en $(t+1)$

$\mathrm{H}_{05}$ : El desempleo en ( $\mathrm{t}$ ) no ayuda a predecir el emprendimiento en $(t+2)$

$\mathrm{H}_{06}$ : El desempleo en $(t+1)$ no ayuda a predecir el emprendimiento en $(t+2)$

Las variables para el año (t) están organizadas en las filas de las matrices de transición, mientras que las variables para los años $(t+1) o ;(t+2)$ en las columnas de las matrices. (1900) es:

El valor del test chi-squared de Pearson

$$
\chi^{2}=\sum_{i=1}^{r} \sum_{j=1}^{c} \frac{\left(O_{i, j}-E_{i, j}\right)^{2}}{E_{i, j}}
$$

donde $O_{i, j}$ es la frecuencia experimental (observada) asociada a la i-ésima clase de frecuencia para el grupo; $E_{i}$ es la frecuencia esperada calculada de la ley de distribución teórica para la i-ésima clase de frecuencia para el grupo $;$; r es el número de niveles de una variable categórica en el año t (número de filas) y c, es el número de niveles en la otra variable categórica en el año ((t+1); número de columnas de las matriz). El número de grados de libertad es igual al número total de celdas $(r c)$ de la matriz, menos la reducción de los grados de libertad, es decir $D F=$ $(r$ - 1)(c-1). El P-value es la probabilidad de observar una estadística muestral tan extrema como el test estadístico. Puesto que el test estadístico es un ChiCuadrado, se usa dicha distribución para calcular la probabilidad asociada con dicho test con los grados de libertad ya indicados.

\section{INFORMACIÓN UTILIZADA}

Del Servicio de Impuestos Internos (SII) para los años 2009 al 2012 se obtiene la información que será empleada. La comuna de pertenencia de cada empresa se determina por la dirección registrada ante el SII como domicilio, o en su defecto por la información entregada por el declarante en la Operación Renta respectiva. Se usó la categorización que tiene SII para 18 actividades económicas (ver anexo, tabla 2). El rubro económico se determina según las actividades que se inscriban al momento de realizar el inicio de actividades por primera vez. Fueron excluidas por reportar muchos casos con valor cero en la variable llamada "Número de Empresas Totales" los sectores económicos: M (Administración Pública y Defensa, Planes de Seguridad Social y Afiliación Obligatoria) y R (Organizaciones y órganos extraterritoriales).

El concepto de emprendimiento es un fenómeno heterogéneo que puede ser interpretado de un modo muy diferente en diferentes contextos. En 
este trabajo se usa como proxy la definición estándar utilizada dada por:

\section{Tasa de Creación de Empresas $(t)(j)(i)=$}

$$
\frac{N^{\circ} \text { de Empresas Nuevas }(t)(j)(i)}{N^{\circ} \text { Empresas Totales }(t)(j)(i)}
$$

donde el número de empresas nuevas corresponde al número de contribuyentes de primera categoría que inician actividades el año (t) para la comuna (j) en el sector económico (i). Un contribuyente se clasifica como empresa si cumple uno o más de los siguientes atributos:

- Es identificado como contribuyente de

\section{1ra Categoría.}

- Presenta declaración jurada 1887 o declaración jurada 1827.

- Es declarante vigente de IVA

Esta clasificación se realiza durante el segundo semestre de cada año. Si un contribuyente presenta su declaración o rectificatoria con nuevos antecedentes posterior a la fecha de clasificación, no queda clasificado.

El número de Empresas Totales (t)(j)(i) es el número total de contribuyentes de primera categoría en el año (t), para la comuna (j) en el sector económico (i), cumpliéndose los mismos criterios detallados anteriormente para los contribuyentes de primera categoría.

Puede notarse que existen a lo menos dos ventajas provenientes de usar tal definición. En primer lugar, si bien no se trata de una medida directa, es una proxy útil de la actividad emprendedora. En segundo lugar, esta medida puede ser reconstruida de forma simple por lo que puede ser comparada a través del tiempo.

La segunda variable es el empleo. Para eso se ha utilizado la siguiente definición de creación de empleo:

Tasa Crecim. Empleo Dependiente $=$

$$
\frac{N^{\circ} \text { Trabajadores Dependientes }(t)(j)(i)}{N^{\circ} \text { Trabajadores Dependientes }(t-1)(j)(i)}
$$

donde el número de trabajadores dependientes ( $\mathrm{t}$ ) (j)(i) es el número total de trabajadores correspon- dientes a todos aquellos trabajadores dependientes informados por sus empleadores en el formulario 1887 para el periodo (t) en la comuna (j) en el sector económico (i), y van asociados a la dirección del domicilio o casa matriz de la empresa. Los trabajadores se contabilizan por empleador, por lo que aquellos con más de una relación de dependencia laboral en el año se cuentan en cada una de ellas. Debe notarse que existe un número importante de recuadros sin informar debido a restricciones relativas a la reserva o secreto tributario (Art. 35 del Código Tributario).

\section{RESULTADOS}

La tabla 3 muestra las estadísticas descriptivas de las series que han sido construidas de acuerdo con el apartado metodológico y; el gráfico 1, representa los resultados en formato tipo "caja" relevantes con medidas de posición y dispersión estadística de los datos. En un gráfico tipo "caja", la parte alta de la caja representa el tercer cuartil (Q3, 75\%); el valor del segundo cuartil se representa en la mediana (50\%) y, la parte inferior de la caja corresponde al primer cuartil (Q1, 25\%). La "cruz" representa la localización de la media de los datos. La altura de la caja representa el rango intercuartil (IQR), es decir la diferencia entre el tercer y el primer cuartil (Q3-Q1). El punto más alto y más bajo fuera de la caja representan un "valor atípico o outlier".

De los gráficos obtenidos puede observarse que a excepción de la serie tasa de desempleo del año 2010, todas las demás series presentan un aparente sesgo hacia la izquierda consistente con el hecho de que se trata de tasas de variaciones (existe un límite inferior mínimo de $-100 \%$, mientras que su límite superior puede ser muy alto). En este caso, bajo el criterio de que los outliers se ubican en $3 \times \mathrm{IQR}$ o más veces por arriba del tercer cuartil (ó $3 \times I Q R$ o más por debajo del primer cuartil), puede sospecharse de la existencia de outliers en las series. Respecto a la distribución, la mayoría de las series cumple con el criterio usual de normalidad de IQR= $1.35 \sigma$, donde $\sigma$ es la desviación estándar de la población.

A partir de las tablas de contingencia obtenidas, se construye la tabla 4a) y 4b) y se estiman 
Tabla 3. Estadísticas Descriptivas.

\begin{tabular}{|c|c|c|c|c|c|c|}
\hline Statistic & $\begin{array}{c}\text { Tasa_ } \\
\text { Empresas2010 }\end{array}$ & $\begin{array}{c}\text { Tasa_ } \\
\text { Empresas2011 }\end{array}$ & $\begin{array}{c}\text { Tasa_ } \\
\text { Empresas2012 }\end{array}$ & $\begin{array}{c}\text { Tasa__ } \\
\text { Empleo2010 }\end{array}$ & $\begin{array}{c}\text { Tasa__ } \\
\text { Empleo2011 }\end{array}$ & $\begin{array}{c}\text { Tasa_ } \\
\text { Empleo2012 }\end{array}$ \\
\hline $\begin{array}{l}\mathrm{N}^{\circ} \text {. de } \\
\text { observaciones }\end{array}$ & 80 & 80 & 80 & 80 & 80 & 80 \\
\hline Mínimo & 0,000 & 0,000 & 0,000 & $-1,000$ & $-0,674$ & $-0,536$ \\
\hline Máximo & 0,439 & 0,472 & 0,527 & 0,761 & 3,481 & 2,300 \\
\hline 1er Cuartíl & 0,040 & 0,042 & 0,042 & $-0,077$ & $-0,005$ & $-0,068$ \\
\hline Mediana & 0,073 & 0,081 & 0,083 & 0,008 & 0,077 & 0,030 \\
\hline 3er Cuartíl & 0,106 & 0,111 & 0,120 & 0,097 & 0,191 & 0,178 \\
\hline Promedio & 0,103 & 0,099 & 0,109 & 0,019 & 0,164 & 0,097 \\
\hline Varianza (n-1) & 0,012 & 0,009 & 0,012 & 0,054 & 0,281 & 0,153 \\
\hline $\begin{array}{l}\text { Desviación } \\
\text { Estándar (n-1) }\end{array}$ & 0,108 & 0,093 & 0,108 & 0,233 & 0,530 & 0,391 \\
\hline Sesgo (Fisher) & 1,954 & 1,962 & 2,067 & $-0,490$ & 4,287 & 3,061 \\
\hline Kurtosis (Fisher) & 3,197 & 4,389 & 4,449 & 5,122 & 23,589 & 14,224 \\
\hline
\end{tabular}

Fuente: Propia.

las pruebas estadísticas de independencia de las pruebas Chi cuadrado en un rango de tiempo de 1 y 2 años. En primer lugar, la tabla 4a) muestra que respecto a un poder explicativo del emprendimiento por sobre el empleo, esto es inexistente ( $p=0,217, p=0,227$ y $p=0,191)$. En efecto, a la significancia habitual del $5 \%$ ninguna de las tres hipótesis de inexistencia del efecto Oportunidad pude ser rechazada.

Por otro lado, la Tabla $4 \mathrm{~b}$ muestra que solamente la hipótesis de una relación Empleo 2011 a Emprendimiento 2012 se mantiene (pvalue $=0.004$ ), permitiendo concluir la existencia de un Efecto Refugio en el caso de un año para un efecto del empleo sobre el emprendimiento. Este resultado indica claramente que la prueba Chi cuadrado apoya la existencia de un Efecto Refugio en la Patagonia.

Puesto que el test Chi cuadrado es una aproximación al Test General de Razón de Máxima

Tabla 4a. Resultados del Test Chi de Independencia para las hipótesis de efecto Oportunidad.

\begin{tabular}{lccc}
\hline & Empre 2010 - Empleo 2011 & Empre 2010- Empleo 2012 & Empre 2011- Empleo 2012 \\
\hline Chi-Cuadrado (valor observado) & 5,767 & 5,644 & 6,113 \\
Chi-square (calor crítico) & 9,488 & 9,488 & 9,488 \\
DF (grados de libertad) & 4 & 4 & 4 \\
valor-p & 0,217 & 0,227 & 0,191 \\
alfa & 0,05 & 0,05 & 0,05 \\
\hline
\end{tabular}

Tabla 4b. Resultados del Test Chi de Independencia para las hipótesis de efecto refugio.

\begin{tabular}{lccc}
\hline & Empleo 2010 - Empre 2011 & Empleo 2010- Empre 2012 & Empleo 2011- Empre 2012 \\
\hline Chi-Cuadrado (valor observado) & 5,857 & 5,228 & 15,436 \\
Chi-square (calor crítico) & 9,488 & 9,488 & 9,488 \\
DF (grados de libertad) & 4 & 4 & 4 \\
valor-p & 0,210 & 0,265 & 0,004 \\
alfa & 0,05 & 0,05 & 0,05 \\
\hline
\end{tabular}


Gráfico 1. Diagramas de caja de las Series.
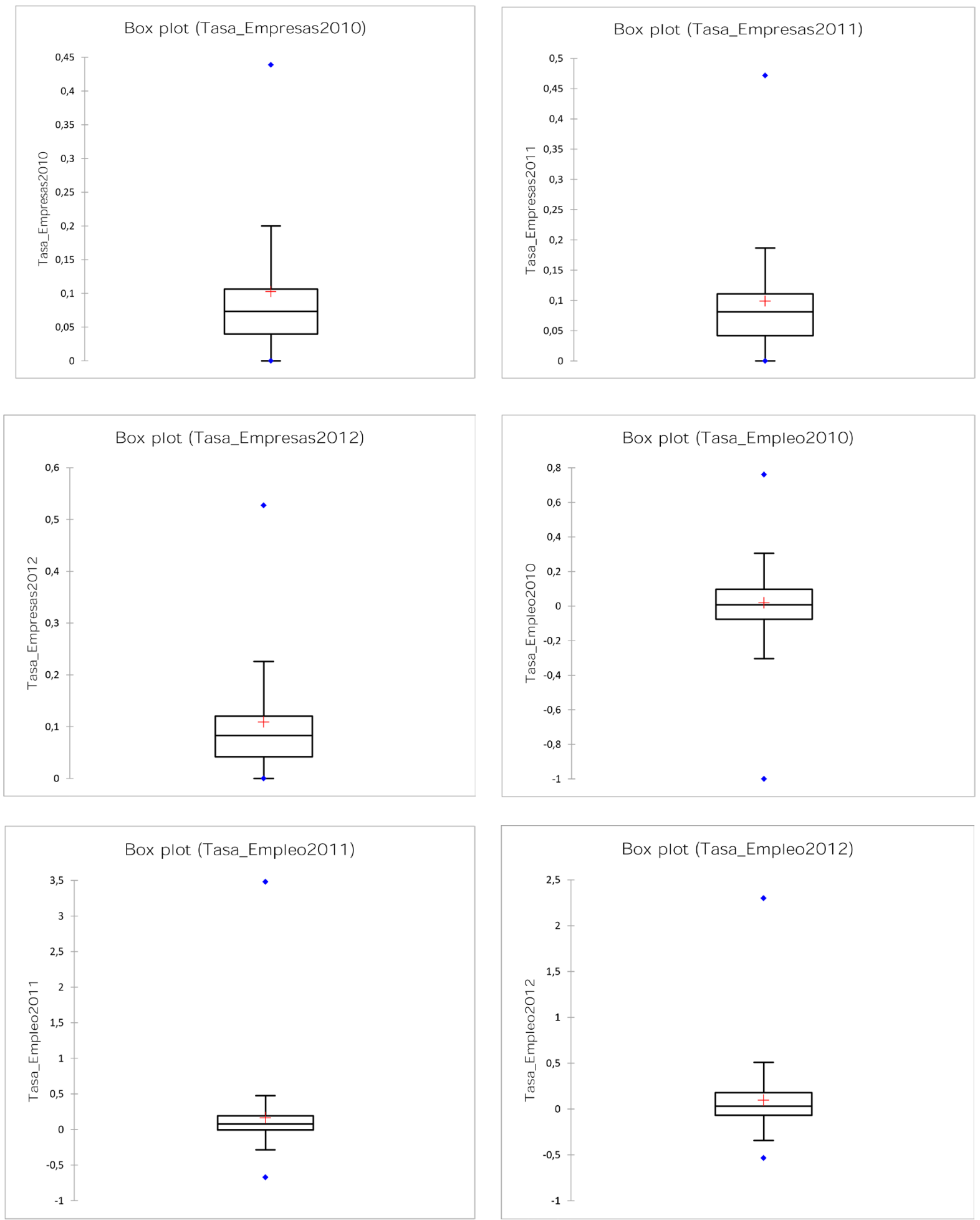

Fuente: Propia. 
Verosimilitud, también ha sido calculado el G-test para corroborar sus resultados, el que queda definido por

$$
G=2 \sum_{i} O_{i} \ln \left(\frac{O_{i}}{E_{i}}\right)
$$

donde $O_{i}$ es la frecuencia observada en una celda de la matriz de transición, y $E_{i}$ es la frecuencia esperada en la hipótesis nula.

Adicionalmente, se ha calculado la prueba exacta de Fisher (Fisher's exact test), la cual es estimada para pequeñas muestras con el fin de examinar la significancia de la asociación entre dos tipos de clasificación (filas versus columnas de la tabla).

La hipótesis nula tanto para el G-test como para la prueba exacta de Fisher viene dada por:

$\mathrm{H}_{0}$ : Las filas y las columnas de la tabla son independientes

La tabla 5 muestra ambos resultados, destacando el hecho que los resultados y principales conclusiones de la tabla 3 se mantienen total- mente. Así por ejemplo, en el caso de la relación Emprendimiento2010-Empleo2011, puesto que el p-value (0.213) es mayor que el nivel de significancia alpha $=0.05$, el test G2 indica que no se puede rechazar la hipótesis nula $\mathrm{H}_{0}$, y que el riesgo de rechazar la hipótesis nula cuando es verdadera es de $19.93 \%$. En el caso del test de razón de verosimilitud, puesto que el $\mathrm{p}$-value calculado (0.213) es mayor que el nivel de significancia alpha $=0.05$, entonces no se puede rechazar la hipótesis nula $\mathrm{H}_{0}$, y el riesgo de rechazar $\mathrm{H}_{0}$ cuando es verdadera es de $21.35 \%$.

En resumen, todas las pruebas realizadas son consistentes en mostrar evidencia de un tipo de emprendimiento basado en una necesidad en la Patagonia Chilena, lo que según la evidencia internacional es característica de los países o zonas con menor nivel de ingresos. Este resultado también es consistente con los hallazgos a favor del Efecto Refugio en Chile encontrados por Hinojosa et al. y por el Ministerio de Economía en 2013. Debe notarse sin embargo que mayoritariamente, de los seis casos analizados, en cinco de ellos no encontramos ninguna relación entre empleo y creación de empresas. Esto también es consistente con mucho de los resultados interna-

Tabla 5. Resultados del Test de Razón de Verosimilitud y la prueba exacta de Fisher para la independencia.

\begin{tabular}{lccc}
\hline & Empre 2010 - Empleo 2011 & Empre 2010- Empleo 2012 & Empre 2011- Empleo 2012 \\
\hline valor-p (dos colas) & 0,213 & 0,231 & 0,203 \\
alfa & 0,05 & 0,05 & 0,05 \\
Wilks' G' (valor observado) & 5,997 & 5,775 & 5,997 \\
Wilks' G' (valor crítico) & 9,488 & 9,488 & 9,488 \\
DF (grados de libertad) & 4 & 4 & 4 \\
valor-p & 0,199 & 0,217 & 0,199 \\
alfa & 0,05 & 0,05 & 0,05 \\
\hline & Empleo 2010 - Empre 2011 & Empleo 2010- Empre 2012 & Empleo 2011- Empre 2012 \\
\hline valor-p (dos colas) & 0,234 & 0,271 & 0,003 \\
alfa & 0,05 & 0,05 & 0,05 \\
Wilks' G' (valor observado) & 5,725 & 5,328 & 16,346 \\
Wilks' G' (valor crítico) & 9,488 & 9,488 & 9,488 \\
DF (grados de libertad) & 4 & 4 & 4 \\
valor-p & 0,221 & 0,255 & 0,003 \\
alfa & 0,05 & 0,05 & 0,05 \\
\hline
\end{tabular}

Fuente: Propia. 


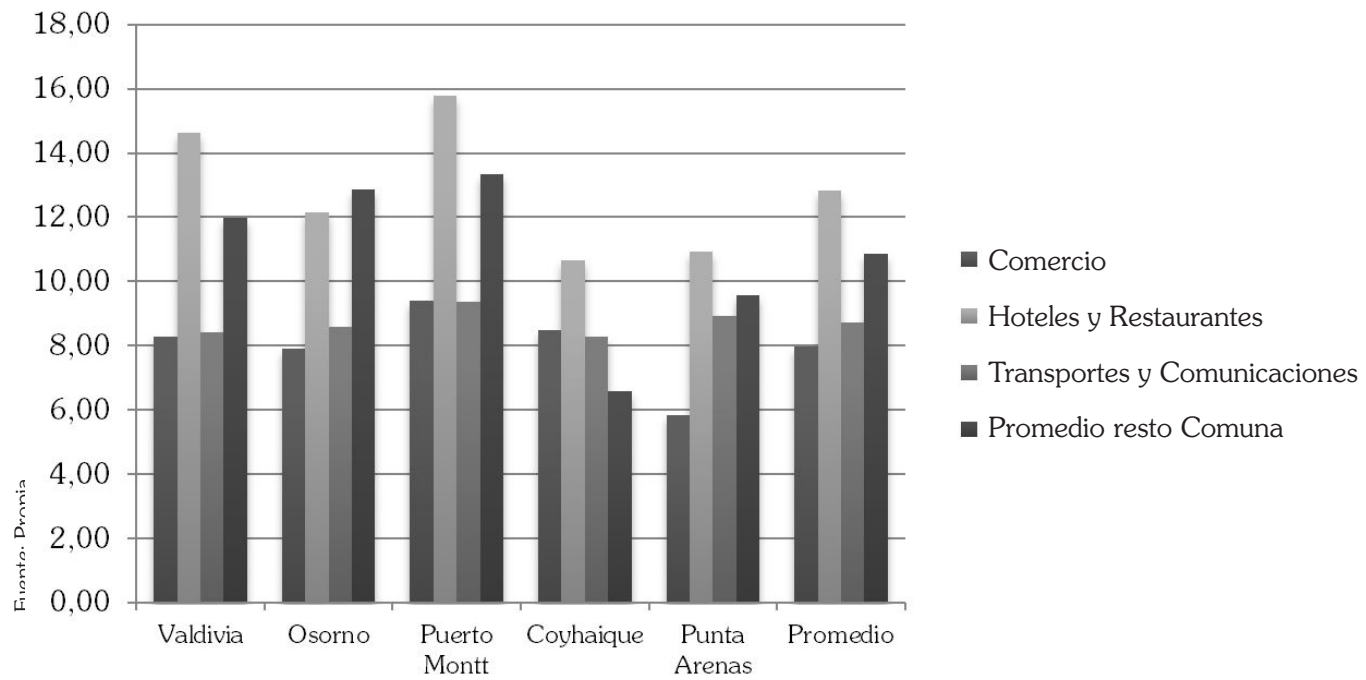

Gráfico 2. Creación de Empresas por Comuna para el Período 2009-12 (en porcentaje).

cionales, en los cuales generalmente se obtienen relaciones muy ruidosas y poco concluyentes, como por ejemplo Carree (2001), Audretsch et al. (2001) y Thurik (2003). El gráfico 2, señala para las comunas de Valdivia, Osorno, Puerto Montt, Coyhaique y Punta Arenas muestra cuál fue durante el período 2009-2012, la creación de empresas ligadas al sector turismo (comercio, restaurantes y hoteles y trasporte), esto respecto al promedio que experimentaron en igual tiempo el resto de las actividades por comuna. En lo particular, se puede observar que, la creación de empresas en el sector turismo es menor que la registrada por el resto del sistema de industrias, excepto en el caso puntual de la comuna de Coyhaique, en donde éste fue mayor y siempre se mostró en constante ascenso respecto a lo que manifestaron el resto de sectores económicos.

En términos de actividad, sólo restaurantes y hoteles, en todas las comunas -exceptuando la de Osorno-, siempre se manifestó por sobre el promedio. Ello obedecería, entre otras razones, a la facilidad de entregar este tipo de servicios en las casas o domicilios particulares. El caso

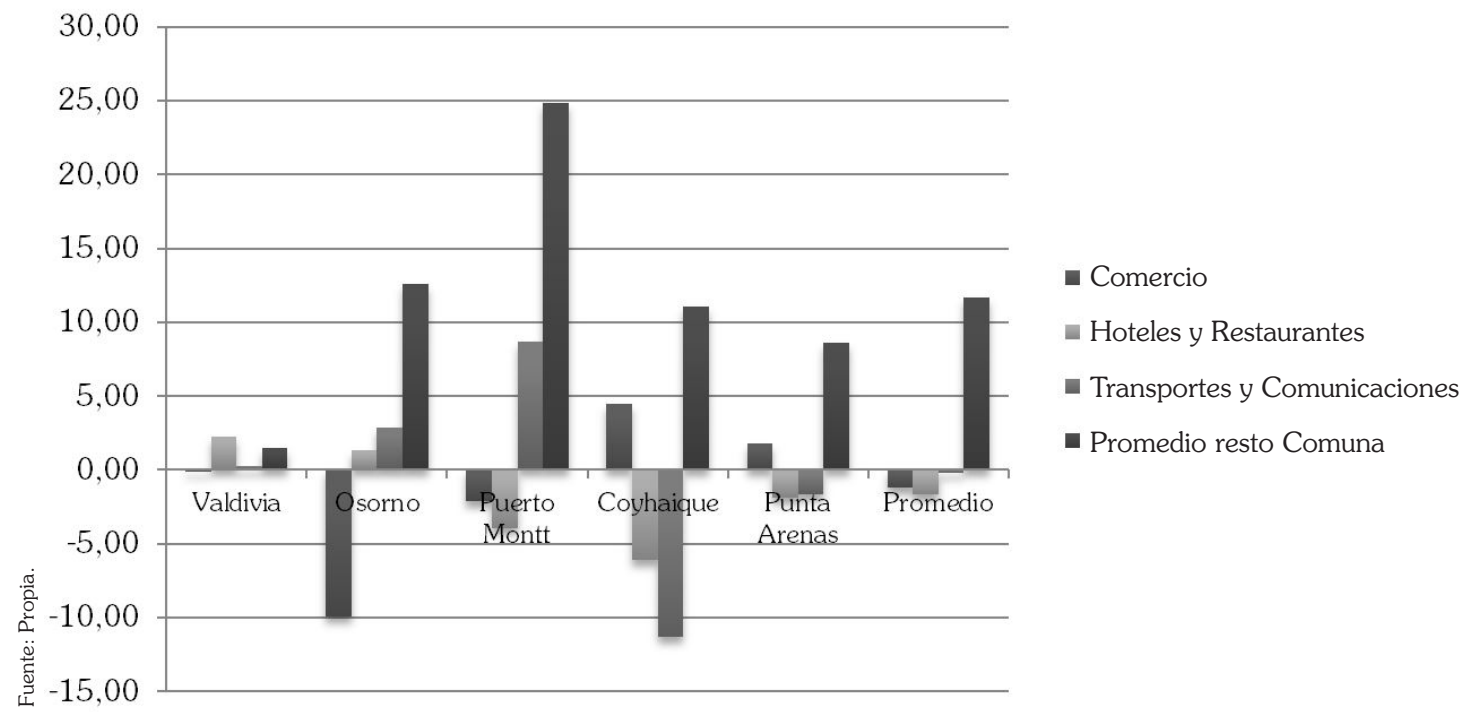

Gráfico 3. Tasa de creación de Empleos por Comuna para el Período 2009-12 (en porcentaje). 
de la comuna de Osorno es particularmente curioso, ya que dicha comuna es un centro nodal próximo a los Parques Nacionales Vicente Pérez Rosales y Puyehue, los cuales según cifras de la Conaf (2012) a ellos durante el año 2012 accedieron cerca de 500.000 personas. En tal sentido se observa que la vocación productiva de ésta comuna dista aún de focalizarse en actividades relacionadas con el turismo. En esta misma línea, para el caso de la Comuna de Punta Arenas, autores como Maripani y Soza-Amigo (2014); Soza-Amigo y Aroca (2012); SozaAmigo y Maripani (2011), destacan que para tal comuna el turismo en términos económicos es muy incipiente no aportando por tanto al desarrollo local, por su parte en Soza-Amigo (2011), se observa una situación similar pero a nivel regional en el sur de Chile.

En el gráfico 3, siguiendo la lógica anterior, se presenta la tasa de generación de empleos para igual período. El gráfico muestra que; en general, en ninguna de las actividades que se podrían vincular al turismo cambian a tasas de Empleo mayores que el promedio de industrias en sus respectivas comunas. Las excepciones son, curiosamente, Hoteles y Restaurantes y Transporte y Comunicaciones en Osorno. Donde se observa una creación de empleos muy por debajo del promedio, mientras en el resto más bien se ve una baja en la generación de estas actividades.

\section{CONCLUSIONES}

A nivel internacional existe abundante literatura tratando de determinar la relación entre desempleo y emprendimiento, el que usualmente ha producido resultados ambiguos. En efecto, mientras que algunos estudios encuentran una relación positiva entre desempleo y emprendimiento (efecto Refugio), otros encuentran evidencia que apoya una relación negativa (efecto Oportunidad). En este estudio se han evidenciado dichos efectos utilizando datos empíricos de las principales comunas de la Patagonia Chilena para los años 2009 a 2012.

Para corroborar lo anterior, se ha aplicado la técnica de matrices de transición, construyendo matrices que capturan la dinámica de la creación de empleos y del emprendimiento, de modo que todos los elementos de la diagonal de estas matrices contienen el número de sectores de las distintas comunas en donde se mantienen el mismo nivel de crecimiento de empleo y de creación de empresas de un periodo a otro. En el estudio se reportan los resultados del estadístico Chi cuadrado y su significancia para cada matriz de transición. Dado que la Hipótesis nula es de independencia de las clasificaciones, se realizaron estimaciones adicionales del G-test basado en pruebas de máxima verosimilitud, y de la prueba exacta de Fisher, con el fin de validar los resultados.

La evidencia encontrada sugiere que la relación entre el desempleo y el espíritu empresarial es difícil de establecer en la Patagonia. Sin embargo en lo que se refiere al periodo 2011-2012 estadísticamente se demuestra que cambios en el desempleo claramente tuvieron un impacto positivo sobre el espíritu empresarial de un año posterior. De acuerdo a esto, el catalizador de la actividad emprendedora es el desempleo, en el sentido de que una situación económica desmejorada impulsa el nacimiento de empresas. La relación inversa, de que cambios en emprendimiento tienen un impacto negativo sobre el desempleo posterior, no ha sido posible de establecer para ninguno de los casos.

El trabajo muestra el importante papel que puede desempeñar el emprendimiento en derribar el desempleo en la Patagonia. Las relaciones empleo y actividad emprendedora son esencialmente dinámicas y heterogéneas, lo cual puede ser la explicación de no encontrar apoyo a las hipótesis planteadas en los otros periodos considerados en el estudio. Puesto que se encuentra aquí apoyo a la hipótesis de que el emprendimiento surge ante una necesidad laboral insatisfecha o limitaciones al trabajo decente, es entonces que surge la necesidad de la población de auto emplearse. De este modo el emprendimiento parece surgir como una respuesta a una dimensión o aspecto negativo, y en este caso las Políticas Públicas que faciliten el emprendimiento sigue siendo un desafío mayor en nuestro país en general, y especialmente en la Patagonia Chilena.

\section{BIBLIOGRAFÍA}

Altman, E., Margaine, M., Schlosser, M. \& Vernimmen, P. 
(1974). Financial and statistical analysis for commercial loan evaluation: a French experience. Journal of Financial and Quantitative Analysis, 195-211.

Alexander, G. \& Chervany, N. (1980). On the Estimation and Stability of Beta. Journal of Financial and Quantitative Analysis, 15(1), 123-137.

Amorós, J. E. \& Poblete, C. (2012). GEM Reporte Nacional de Chile. Global Entrepreneurship Monitor. http:// negocios.udd.cl/gemchile/estudios-y-publicaciones/ reportes-nacionales/

Amorós, J. \& Echecopar, G. (2008). Emprender en Chile: ¿Estamos en el camino correcto?. En P. Cortés Durán (Ed.), Emprendimiento e Innovación en Chile. Una Tarea Pendiente, Capítulo III . Santiago: Ediciones Universidad del Desarrollo.

Asociación de Emprendedores de Chile. (2014). Entrevista. http://asech.cl/juan-pablo-swett-el-emprendimiento-por-necesidad-se-da-mucho-cuando-la-economiaesta-mal/

Audretsch, D. (1995). Innovation and Industry Evolution. Cambridge, Mass. MIT Press.

Audretsch, D. \& Thurik, A. (2000). Capitalism and Democracy in the $21^{\text {st }}$ Century: from the Managed to the Entrepreneurial Economy. Journal of Evolutionary Economics, 10, 17-34.

Blanchflower, D. \& Meyer, B. (1994). A Longitudinal Analysis of Young Entrepreneurs in Australia and the United States. Small Business Economics, 6(1), 1-20.

Blau, D. (1987). A Time Series Analysis of Self Employment in the United States. Journal of Political Economy, 95(3), 445-467.

Carree, M. (2001). Does Unemployment Affect the Number of Establishments? A Regional Analysis for U.S. States. Regional Studies. The Journal of the Regional Studies Association, 36(4), 389-398.

Corporación Nacional Forestal (2012). Estadísticas de Visitación SNASPE; disponible en: http://www.conaf.cl/ parques-nacionales/visitanos/estadisticas-de-visitacion/; Accesado el 17 de abril de 2014.

Corfo (2013). Emprendimiento en Chile: Hacia un Nuevo Modelo de Segmentación y Análisis. Unidad de Estudios, Gerencia de Estrategia y Estudio CORFO. Emprendimiento En Chile: Hacia Un Modelo De Segmentación. Corporación de Fomento de la Producción, Corfo.

Evans, D. \& Jovanovic, B. (1989). Estimates of a Model of Entrepreneurial Choice under Liquidity Constraints. Journal of Political Economy, 97(3), 657-674.

Evans, D. \& Leighton, L. (1990). Small Business Formation by Unemployed and Employed Workers. Small Business Economics, 2(4), 319-330.

Evans, D. \& Leighton, L. (1989). The Determinants of Changes in U.S. Self-Employment, 1968-1987. Small Business Economics, 1(2), 111-120.

Hinojosa, S. \& Pardo, C. (2013). Ganas de Emprender y Felicidad: un Estudio Exploratorio a Partir del Global Entrepreneurship Monitor en Chile. Journal of Technology Management \& Innovation, 8(1), 76-89.

Larroulet, C. \& Ramírez, M. (2007). Emprendimiento: Factor clave para la nueva etapa de Chile Estudios Públicos, 108 (primavera 2007), 93-116.

Maripani, J. \& Soza-Amigo, S. (2014). Turismo en Chile, Una Estimación de su Demanda y sus Efectos en el PIB: El caso de la Patagonia Austral. Gestión y Política Pública, Mimeo.

Ministerio de Economía (2013). Encuesta de Microemprendimiento. Emprendimiento Formal e Informal en Chile Análisis a partir de los resultados de la Tercera Encuesta de Microemprendimiento 2013. División de Estudios Ministerio de Economía.

Pearson, K. (1900). On the criterion that a given system of deviations from the probable in the case of a correlated system of variables is such that it can be reasonably supposed to have arisen from random sampling. Philos. Mag. 1900, 50, 157-175.

Picot, G., Manser, M. \& Lin, Z. (1998). The Role of Self-Employment in Job Creation In Canada and the U.S. OECD-CERF-CILN International Conference on Self-Employment, Burlington, Ontario, Canada.

Reynolds, P., Miller, B. \& Maki, W. R. (1995). Explaining Regional Variation in Business Births and Deaths: U.S. 1976-1988. Small Business Economics, 7 (5), 389-707.

Ricardo, D. (1821, 1ra edición) 1951. On the Principles of Political Economy and Taxation. P. Sraffa (Ed.). Cambridge: University Press.

Thurik, R. (2003). Entrepreneurship And Unemployment. In The UK. Scottish Journal of Political Economy, 50(3), 264-290.

Schumpeter, J. A. (1942, 1ra edición) 1952. Capitalism, Socialism and Democracy. 5th ed. London: George Allen \&Unwin

Servicio de Impuestos Internos de Chile (SII). Información preparada por el Área de Información y Estadísticas Tributarias del Departamento de Estudios Económicos y Tributarios, Subdirección de Estudios SII, disponible en: http://www.sii.cl/estadisticas/

Soza-Amigo, S. \& Aroca, P. (2012). Simulation and Analy- 
sis of Punta Arenas Economy Based on Input-Output Tables 2003 and 2007. Magallania, 40(2), 113-127. Soza-Amigo, S. \& Maripani, J. (2011). The Input-Output Matrix of the Punta Arenas City: Description, analysis and simulation. Magallania, 39(1),103-118.

Soza-Amigo, S. (2011). Linkages and Structural Similarities for the Regions of Chile. Economic Analysis Review,
26(2), 81-110.

Storey, D. (1991). The Birth of New Firms-Does Unemployment Matter? A Review of the Evidence. Small Business Economics, 3(3), 167-178.

Theobald, M. (1981). Beta Stationarity and Estimation Period: Some Analytical Results. Journal of Financial and Quantitative Analysis, 16(5), 747-757.

\section{ANEXO}

Tabla 2. Clasificación de Actividades Económicas utilizadas por el SII
A - Agricultura, ganadería, caza y silvicultura
B - Pesca
C - Explotación de minas y canteras
D - Industrias manufactureras no metálicas
E - Industrias manufactureras metálicas
F - Suministro de electricidad, gas y agua
G - Construcción
$\mathrm{H}$ - Comercio al por mayor y menor, reparación de vehícu-
los automotores/enseres domésticos
I - Hoteles y restaurantes
$\mathrm{J}$ - Transporte, almacenamiento y comunicaciones

$\mathrm{K}$ - Intermediación financiera

L - Actividades inmobiliarias, empresariales y de alquiler M - Administración Pública y defensa, planes de Seguridad Social afiliación obligatoria

$\mathrm{N}$ - Enseñanza

O - Servicios sociales y de salud

$\mathrm{P}$ - Otras actividades de servicios comunitarias, sociales y personales

$\mathrm{Q}$ - Consejo de administración de edificios y condominios

$\mathrm{R}$ - Organizaciones y órganos extraterritoriales

Fuente: Servicios de Impuestos Internos (SII). 
